\title{
Distinct approaches to language pathway tractography: comparison of anatomy-based, repetitive navigated transcranial magnetic stimulation (rTMS)-based, and rTMS-enhanced diffusion tensor imaging-fiber tracking
}

\author{
Luca L. Silva, MD, ${ }^{1,2}$ Mehmet S. Tuncer, ${ }^{1}$ Peter Vajkoczy, MD, ${ }^{1}$ Thomas Picht, MD, ${ }^{1,3}$ and \\ Tizian Rosenstock, MD ${ }^{1,4}$
}

Departments of ${ }^{1}$ Neurosurgery and ${ }^{2}$ Anesthesiology and Intensive Care Medicine, Charité - Universitätsmedizin Berlin, corporate member of Freie Universität Berlin and Humboldt-Universität zu Berlin; ${ }^{3}$ Cluster of Excellence: "Matters of Activity. Image Space Material"-Humboldt University, Berlin; and "Berlin Institute of Health $(\mathrm{BIH})$ at Charité - Universitätsmedizin Berlin, Germany

OBJECTIVE Visualization of subcortical language pathways by means of diffusion tensor imaging-fiber tracking (DTIFT) is evolving as an important tool for surgical planning and decision making in patients with language-suspect brain tumors. Repetitive navigated transcranial magnetic stimulation (rTMS) cortical language mapping noninvasively provides additional functional information. Efforts to incorporate rTMS data into DTI-FT are promising, but the lack of established protocols makes it hard to assess clinical utility. The authors performed DTI-FT of important language pathways by using five distinct approaches in an effort to evaluate the respective clinical usefulness of each approach.

METHODS Thirty patients with left-hemispheric perisylvian lesions underwent preoperative rTMS language mapping and DTI. FT of the principal language tracts was conducted according to different strategies: la, anatomical landmark based; Ib, lesion-focused landmark based; Ila, rTMS based; Illb, rTMS based with postprocessing; and III, rTMS enhanced (based on a combination of structural and functional data). The authors analyzed the respective success of each method in revealing streamlines and conducted a multinational survey with expert clinicians to evaluate aspects of clinical utility.

RESULTS The authors observed high usefulness and accuracy ratings for anatomy-based approaches (la and lb). Postprocessing of rTMS-based tractograms (Illb) led to more balanced perceived information content but did not improve the usefulness for surgical planning and risk assessment. Landmark-based tractography (la and lb) was most successful in delineating major language tracts ( $98 \%$ success), whereas rTMS-based tractography (Ila and IIb) frequently failed to reveal streamlines and provided less complete tractograms than the landmark-based approach $(p<0.001)$. The lesionfocused landmark-based (Ib) and the rTMS-enhanced (III) approaches were the most preferred methods.

CONCLUSIONS The lesion-focused landmark-based approach (Ib) achieved the best ratings and enabled visualization of the principal language tracts in almost all cases. The rTMS-enhanced approach (III) was positively evaluated by the experts because it can reveal cortico-subcortical connections, but the functional relevance of these connections is still unclear. The use of regions of interest derived solely from cortical rTMS mapping (Ila and Ilb) leads to cluttered images that are of limited use in clinical practice.

https://thejns.org/doi/abs/10.3171/2020.12.JNS204028

KEYWORDS brain tumor surgery; DTI fiber tracking; tractography; language network; TMS/rTMS; repetitive navigated transcranial magnetic stimulation; arcuate fascicle; diagnostic technique

$\mathrm{B}$ RAIN tumors in proximity to so-called eloquent regions pose a major challenge to the neurosurgical discipline, as their resection is associated with an increased risk of postoperative functional deficits. This situation underlines the unambiguous need for reliable predictive tools to locate language function areas in the brain to aid decision-making processes of surgeons and patients. According to the commonly taught Broca-Wernicke

ABBREVIATIONS AF = arcuate fascicle; $C C=$ contingency coefficient; $D C S=$ direct cortical stimulation; DTI-FT = diffusion tensor imaging-fiber tracking; FA = fractional anisotropy; FAT = frontal aslant tract; IFOF = inferior fronto-occipital fascicle; ILF = inferior longitudinal fascicle; $n$ TMS = navigated TMS; ROI = region of interest; rTMS = repetitive $\mathrm{nTMS} ; \mathrm{SMD}=$ standardized mean difference; $\mathrm{TMS}=$ transcranial magnetic stimulation; UF = uncinate fascicle; la = anatomical landmark based; Ib = lesionfocused landmark based; Ila = rTMS based; Ilb = rTMS based with postprocessing; III = rTMS enhanced (based on a combination of structural and functional data). SUBMITTED November 12, 2020. ACCEPTED December 21, 2020.

INCLUDE WHEN CITING Published online July 30, 2021; DOI: 10.3171/2020.12.JNS204028. 
model of cortical speech organization, language function is harbored in two principal cortical areas with respective involvement in articulatory (Broca's area) and semantic (Wernicke's area) aspects. ${ }^{1}$ Advancements in the field of neurobiology, however, reveal that this simplistic model does not adequately represent the distributed connectivity involved in language. More-contemporary models such as the dual-stream model of language proposed by Hickok and Poeppel have thus largely replaced prior concepts. ${ }^{2}$ Evidence suggests that a largely bihemispheric ventral stream processes speech signals for comprehension, and a predominantly left-hemispheric dorsal stream maps acoustic speech signals to frontal lobe articulatory networks. ${ }^{2}$ The integrity of white matter tracts connecting perisylvian cortical areas has been shown to be essential for intact language function. ${ }^{3,4}$ As the understanding of language functional anatomy evolves, diagnostic strategies for neurosurgical patients must be adapted accordingly. ${ }^{5}$ Electrical direct cortical stimulation (DCS) during awake surgery has remained the gold standard for mapping cortical language function for several decades. ${ }^{6}$ Novel noninvasive methods such as repetitive navigated transcranial magnetic stimulation (rTMS) have emerged and are being intensively studied for their ability to interrogate language pathways. Ample evidence suggests a correlation between rTMS-based and DCS-based maps of cortical language function, concluding with positive predictive values of $16 \%-69 \%$ and negative predictive values of $83.9 \%-99 \% .^{7-9}$ Consequently, it was shown that rTMS-based cortical maps aid the preservation of functional cortical areas by providing reliable "negative mappings" during tumor surgery due to their high sensitivity.$^{10}$ Cortical mappings alone, however, cannot reveal crucial subcortical language pathways. Diffusion tensor imaging-fiber tracking (DTI-FT) is a technique that provides information on the architecture of subcortical structures, thereby allowing for noninvasive preoperative tractography of white matter tracts. Delineation of specific white matter tracts demands the definition of at least one region of interest (ROI). While published methodologies for language pathway tractography are highly heterogeneous, they commonly involve placement of ROIs based on structural landmarks. ${ }^{11}$ Alternatively, a variety of protocols have been reported for placement of ROIs based on functional data from rTMS mappings, but with the same purpose of facilitating preoperative planning and improving patient outcomes. ${ }^{4,12,13}$ A comparative analysis examining individual feasibilities, strengths, and weaknesses of several existing concepts for integration of rTMS and DTI-FT into clinical practice is pending. In an attempt to evaluate and assess the multitude of approaches, we carried out and compared five distinct strategies, with and without the incorporation of functional data from rTMS. We assessed the respective success of these strategies in revealing streamlines in the obligatory anatomical pathways of five principal language tracts and investigated aspects of clinical utility in a multinational survey with 18 expert clinicians.

\section{Methods}

\section{Patients and Study Design}

The published guidelines on standards for reporting on qualitative research were followed in the planning and implementation of this work. ${ }^{14}$ The study was designed as a prospective case collection study. Patients admitted between 2014 and 2017 to the Department of Neurosurgery at Charité - Universitätsmedizin Berlin for tumor removal were screened for enrollment. Inclusion criteria were existence of a brain tumor compressing and/or infiltrating areas presumably involved in language function, age older than 18 years, and written informed consent. Exclusion criteria were existence of intracranial implants and clinically severe aphasia hindering successful completion of the object-naming task needed for rTMS mapping..$^{15}$ Preoperative aphasia grading of all patients was carried out using four subtests from the Aachener Aphasia Test (AAT): ${ }^{16}$ verbal repetition, object naming, language comprehension, and the Token Test. Aphasia was classified according to the test score as none (350-310), mild (309280), intermediate (279-230), or severe (229-0). Enrolled patients underwent preoperative MRI as set forth below. Subsequently, functional mapping of the cortical areas was conducted by rTMS. Postoperative aphasia grading was carried out 3-5 days after surgery. All patients were right-handed according to the Edinburgh Handedness Inventory. ${ }^{17}$ This study was carried out in accordance with the recommendations of the responsible Ethics Commission (reference EA4/007/06). Written informed consent was obtained from all patients in accordance with the Declaration of Helsinki.

\section{MRI Acquisition}

High-resolution MRI was carried out on a 3T Skyra scanner (Siemens Healthcare) according to our clinic standard. ${ }^{11}$ The protocol included T1-weighted, contrastenhanced, 3D multiplanar reconstruction (MPR), T2weighted, FLAIR, and DTI sequences (TE $95 \mathrm{msec}$, TR 7.500 , resolution $2 \mathrm{~mm}$ isotropic, 60 directions, $\mathrm{b}=1300$ seconds $/ \mathrm{mm}^{2}$ ). The T1-weighted sequences (TE $2.32 \mathrm{msec}$, TR 2.300, resolution $1 \mathrm{~mm}$ isotropic, flip angle $8 \mathrm{~mm}, \mathrm{~b}=$ 1300 seconds $/ \mathrm{mm}^{2}$ ) were always used as the reference anatomical examination. In case of non-contrast-enhancing lesions, FLAIR and T2-weighted fast spin-echo sequences were used to define tumor margins.

\section{rTMS Language Mapping}

Cortical language mapping of the left hemisphere was performed using the Nexstim eXimia NBS system (version 4.3, Nexstim Oy) with a biphasic figure-eight coil. Initially, the individual cortical excitability threshold was identified by determining the resting motor threshold (RMT) of the first dorsal interosseous (FDI) muscle based on the Rossini-Rothwell criterion. ${ }^{18,19}$ Subsequently, a baseline object-naming task (NexSpeech module, Nexstim Oy) was conducted three times in order to eliminate unrecognized and misnamed objects and minimize falsepositive results. Pictures were presented to the patient for 1 second at an individualized picture-to-picture interval of 2.5-4 seconds. During mapping, rTMS was triggered at the moment of picture presentation without onset delay. The stimulation protocol comprised a train of 5 pulses at $5-\mathrm{Hz}$ frequency at an intensity of $100 \%$ RMT. A total of 

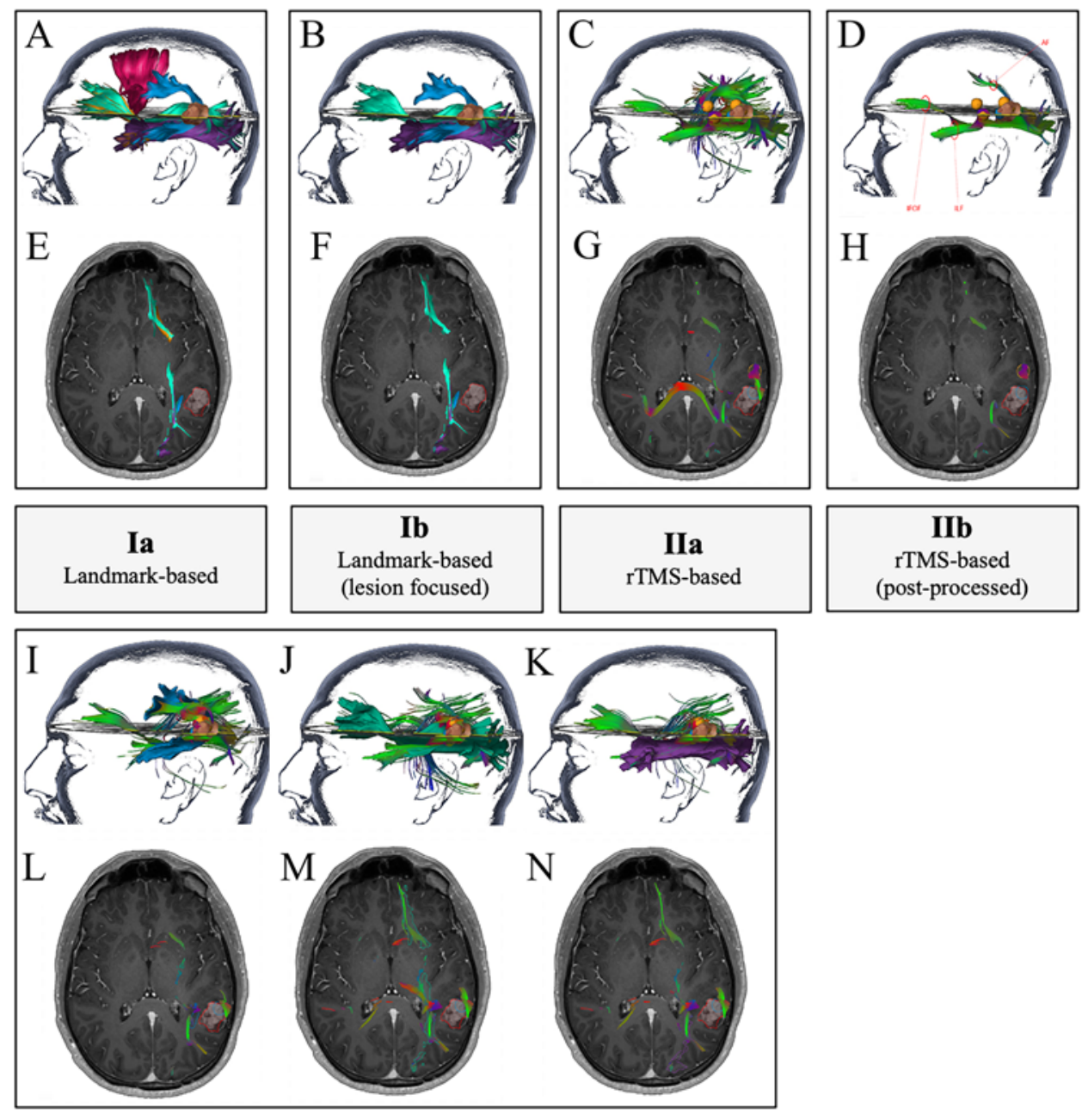

III

rTMS-enhanced

FIG. 1. Exemplary case of a left-sided glioblastoma located in the temporo-parieto-occipital junction. Different tracking algorithms $(\mathrm{la}, \mathrm{lb}, \mathrm{Ila}, \mathrm{Ilb}$, and III) of the five major language fiber bundles (arcuate fascicle = blue, frontal aslant tract = red, inferior fronto-occipital fascicle $=$ green, inferior longitudinal fascicle $=$ purple , and uncinate fascicle $=$ orange) are presented. $\mathbf{A}-\mathbf{D}$ and I-K: Lateral views of $3 \mathrm{D}$ reconstruction of the skull. E-H and L-N: T1-weighted axial slice with contrast agent. Tumor volume is shown in orange and labeled red in the axial slice. The spheres represent enlarged rTMS-positive spots (radius $=5 \mathrm{~mm}$ ). Figure is available in color online only.

$80-120$ sites located in the frontal, temporal, and parietal cortex were stimulated three times each. We followed a recent international consensus paper, where the mapping protocol is described in detail. ${ }^{20}$ Response classification was carried out by two trained and experienced examiners, with at least 5 years of experience and 100 analyzed language mappings, by comparing video recordings of baseline performance with each recorded response during stimulation. Disturbances were classified as positive spots and categorized into four error groups: no response, semantic paraphasia, performance error, and hesitation. In unclear cases, a linguist was consulted to aid the classification process. Language-positive spots were imported into the clinical planning software using the DICOM standard for subsequent segmentation and enlargement.

\section{DTI-FT of Major Language Pathways}

An exemplary overview of tractograms resulting from each method can be found in Fig. 1. The server-based planning software Elements (Brainlab AG) was used for DTI-FT of language tracts. T1 MPRAGE MR images were coregistered with diffusion MR images via the software's automated process after application of the proprietary cranial distortion correction module. ${ }^{21}$ The fractional anisotropy (FA) was computed via the software's deterministic FACT and TEND algorithms. ${ }^{22}$ Five major language tracts were defined: the arcuate fascicle (AF), frontal aslant tract (FAT), inferior fronto-occipital fascicle (IFOF), inferior longitudinal fascicle (ILF), and uncinate fascicle (UF). In the structural approach (Ia), ROIs 
A

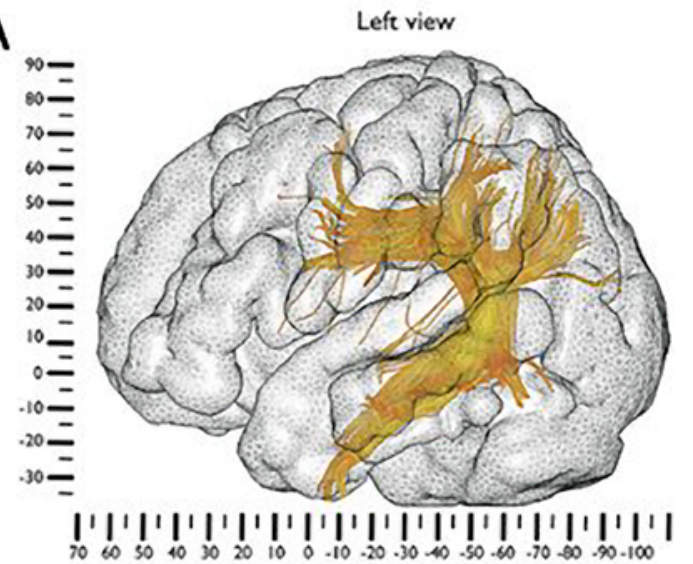

C

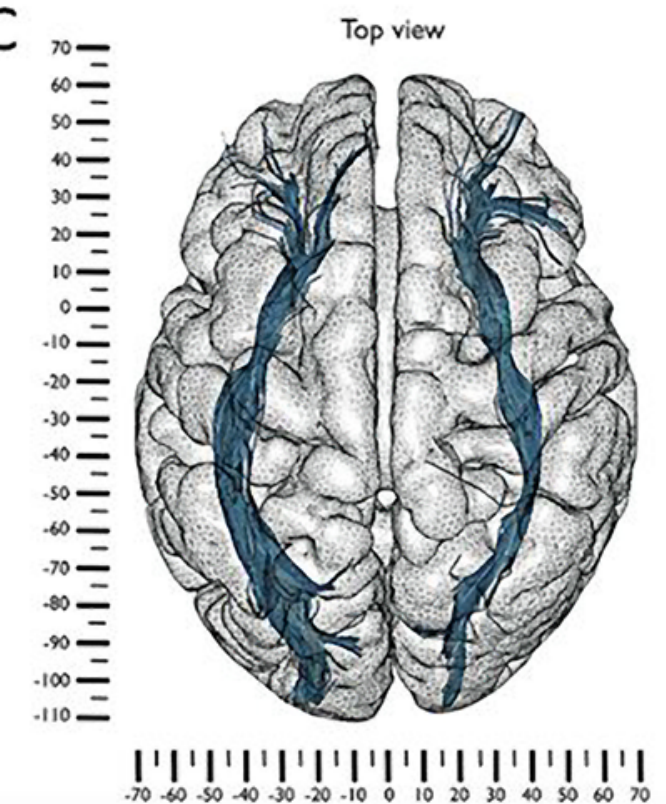

B

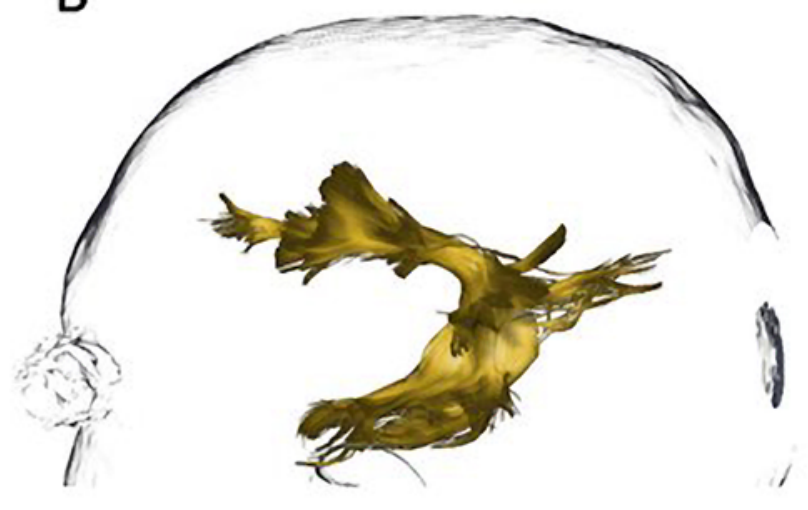

D

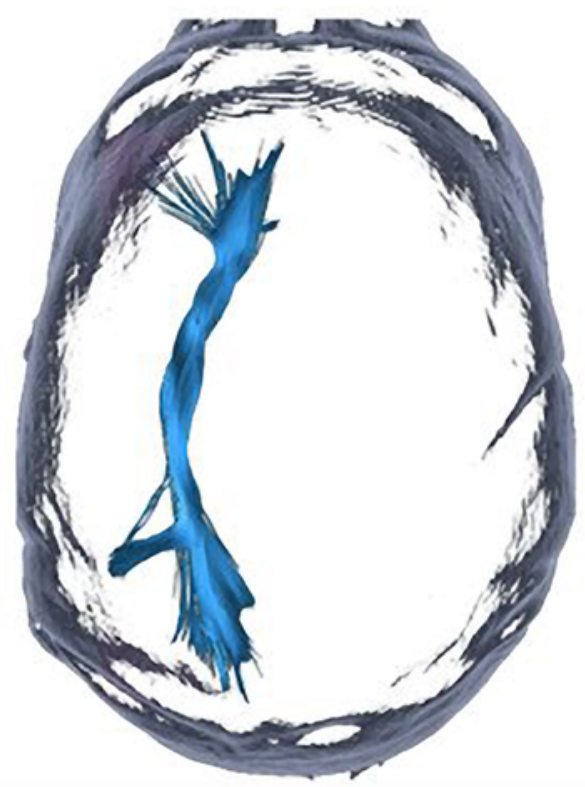

FIG. 2. Exemplary comparison of the tractography atlas from Catani and Thiebaut de Schotten and our landmark-based tractography of the AF and IFOF. A: Lateral view on the AF of the tractography atlas. B: Exemplary lateral view of landmark-based tractography of the AF. C: Top view of the IFOF of the tractography atlas. D: Exemplary top view of the landmark-based tractography of the IFOF. Panels A and C: Reprinted from Cortex, vol 44(8), Catani M, Thiebaut de Schotten M. A diffusion tensor imaging tractography atlas for virtual in vivo dissections, pp 1105-1132, copyright 2008, with permission from Elsevier. Figure is available in color online only.

were manually placed based on established, easy-to-identify anatomical landmarks in the tract obligatory pathways. ${ }^{11}$ All tracking results were verified by the published atlas of Catani and Thiebaut de Schotten (Fig. 2). ${ }^{23}$ The lesion-focused modification (Ib) was aimed at reducing irrelevant information by removal of tractograms with tumor distances greater than $20 \mathrm{~mm}$. The rTMS-based approach (IIa) consisted of the definition of all enlarged rTMS language-positive spots as a single ROI (radius $=5$ $\mathrm{mm})$. Aberrant fibers that were not located in the obligatory anatomical pathways of major language tracts were manually removed during postprocessing (IIb). The combined approach (III) consisted of definition of individual landmark-based tractograms from method $\mathrm{Ib}$, as well as individual rTMS-positive language spots located below the projected craniotomy $(<20 \mathrm{~mm})$ as ROIs. See Table 1 for an overview of parameters used. Volumes of tumors and tractograms were calculated using the software's integrated object creation tool. rTMS-based tractogram completeness was determined based on volume comparisons between landmark-based tractograms and rTMS-based tractograms of the same tract. Volumes were considered low ( $1 \%-33 \%$ of volume), intermediate (33\%-66\% of volume), or high (66\%-100\% of volume).

\section{Expert Evaluation}

Resulting images were provided to 18 experienced neurosurgeons for review either by granting access to the planning software or by providing figures with left lateral views on 3D reconstructions along with axial slices at tu- 
TABLE 1. Tractography approaches and parameters used

\begin{tabular}{|c|c|c|c|c|}
\hline & Description & ROls & DTI-FT Parameters & Postprocessing \\
\hline la & Landmark based & \multirow{2}{*}{ Anatomical landmarks } & \multirow{2}{*}{$\begin{array}{l}\text { FA } 0.1-0.15 \\
\text { MFL } 50 \mathrm{~mm}\end{array}$} & - \\
\hline $\mathrm{lb}$ & Landmark based (lesion focused) & & & Removal of tracts $w /$ tumor distance $>20 \mathrm{~mm}$ \\
\hline Ila & rTMS based & \multirow{2}{*}{$\begin{array}{l}\text { rTMS language spots } \\
\quad(\text { radius }=5 \mathrm{~mm})\end{array}$} & \multirow{2}{*}{$\begin{array}{l}\text { FA } 0.15 \\
\text { MFL } 50 \mathrm{~mm}\end{array}$} & - \\
\hline Ilb & rTMS based (postprocessed) & & & $\begin{array}{l}\text { Removal of aberrant fibers outside of } \\
\text { anatomical pathways }\end{array}$ \\
\hline III & rTMS enhanced & $\begin{array}{l}\text { Landmark-based tractograms in } \\
\text { tumor vicinity (Ib) + rTMS lan- } \\
\text { guage spots (Ila) below projected } \\
\text { craniotomy }\end{array}$ & $\begin{array}{l}\text { FA } 0.12 \\
\text { MFL } 50 \mathrm{~mm}\end{array}$ & - \\
\hline
\end{tabular}

MFL = minimum fiber length.

mor height (Fig. 2). The evaluators comprised 4 professors, 4 consultants, and 10 senior residents (with at least 2 years of experience in glioma surgery) across eight academic centers in Europe and North America. To limit time constraints, most evaluators received 5 semirandomly selected cases representing different tumor locations (2 temporal, 2 frontal, 1 parietal). All clinician evaluators had at least 2 years of experience in DTI-FT-assisted tumor surgery. For each case, raters provided single-choice responses in the following categories: usefulness for surgical planning, usefulness for risk assessment, overall information content, perceived accuracy of visualized tracts, and overall preferred method for each case. The evaluators were not blinded to the technique, as awareness of the method forms an important component of actual clinical utility and decision making. ROI seeding remains visible in all representations incorporating rTMS data. A full evaluation sheet can be found in the Supplemental Material.

\section{Statistical Analysis}

All statistical analyses were conducted using SPSS Statistics, version 25 (IBM Corp.). We used descriptive statistics as well as contingency coefficients (CCs) and standardized mean differences (SMDs) to indicate the sample size-independent magnitude of group differences. Pearson's chi-square test, two-sample t-test, or Mann-Whitney U-test was used depending on the scaling and distribution of the variables. Correlation analysis was performed using Pearson's correlation coefficient. Results with $p<0.05$ were considered statistically significant.

\section{Results}

\section{Patient Characteristics}

Overall, 30 patients with left-sided perisylvian brain lesions were included in the study (Table 2). The population comprised 16 men (53.3\%) and 14 women (46.7\%), with a mean age of $47 \pm 14$ years (range $21-69$ years). The most common tumor entities were glioma WHO grade III (33\%) and glioblastoma WHO grade IV (20\%). Four patients $(13.3 \%)$ suffered from mild aphasia before rTMS mapping. Median tumor volume was $13.3 \pm 20.9 \mathrm{~cm}^{3}$ (range 0.4-74.1 $\mathrm{cm}^{3}$ ). Tumor volume was larger in aphasic patients than in nonaphasic patients $\left(8.6 \mathrm{vs} 37.0 \mathrm{~cm}^{3}\right.$; SMD $=1.01 ; \mathrm{p}=0.051$ ).

\section{rTMS Language Mapping}

Left-hemispheric rTMS language mapping took on average 65 minutes (range 40-95 minutes). Mappings were successfully carried out in all enrolled patients, with no persistent adverse effects. A median of $8.5 \pm 5$ languagepositive spots were identified in each patient (range 2-22), while hesitation was the most frequent error category (Table 2). In total, 116 of 276 spots ( $42 \%$ ) were located below the projected craniotomy (Fig. 3A). The higher the baseline error rate, the higher was the number of languagepositive spots (Pearson's $r=0.361, p=0.050$ ).

\section{DTI-FT Methods}

\section{Landmark Based (la and lb)}

DTI-FT based on anatomical landmarks took on average 24 minutes (range 15-34 minutes). In 28 cases (93\%), successful delineation of all five major language tracts was achieved. In one case the landmark-based approach failed to reveal any streamlines of the UF and in another case the UF and IFOF could not be visualized. Overall, the resulting tractograms correlated well with fiber trajectories found in anatomical atlases (Fig. 1). Eighty-three of 147 tractograms $(56.5 \%)$ were located in proximity $(<20$ $\mathrm{mm}$ ) to the tumor, with AF occurring most frequently and UF least frequently near the tumor (Fig. 3B).

\section{rTMS Based (lla and Ilb)}

DTI-FT based purely on positive rTMS spots took on average 27 minutes (range 19-36 minutes). This technique was not capable of reproducibly visualizing the major language tracts. In comparison to the landmark-based approach, 1 of 5 tracts could be revealed in the median (Table 3; SMD = 4.49; $\mathrm{p}<0.001$ ). This was most evident in the following tracts: UF (0/28), ILF (7/30), and AF (7/30). Furthermore, volumes of all visualized tracts were significantly lower than the volumes of tracts visualized with landmark-based tractography (Ia and Ib) (Fig. 3C). To obtain FT results without aberrant fibers outside of anatomical pathways (IIb), postprocessing was necessary in each case of the rTMS-based approach (IIa).

\section{rTMS Enhanced (III)}

rTMS-enhanced tracking took on average 60 minutes (range 50-69 minutes). Of all possible ROI combina- 
TABLE 2. Patient, tumor, and rTMS mapping characteristics

\begin{tabular}{|c|c|c|c|c|c|c|c|c|c|c|c|c|}
\hline $\begin{array}{r}\text { Pt } \\
\text { No. }\end{array}$ & $\begin{array}{l}\text { Age } \\
\text { (yrs) }\end{array}$ & Sex & $\begin{array}{l}\text { WHO } \\
\text { Grade }\end{array}$ & $\begin{array}{l}\text { Vol } \\
\left(\mathrm{cm}^{3}\right)\end{array}$ & Location & $\begin{array}{c}\text { Preop } \\
\text { Aphasia }\end{array}$ & $\begin{array}{l}\text { Baseline } \\
\text { Error Rate }\end{array}$ & NR & $\mathrm{P}$ & S & $\mathrm{H}$ & Total \\
\hline 1 & 67 & $\mathrm{~F}$ & Glioma IV & 1.1 & Frontal & No & 13.75 & 0 & 2 & 0 & 5 & 7 \\
\hline 2 & 33 & $\mathrm{~F}$ & Glioma III & 28.5 & Opercular & No & 10 & 0 & 1 & 0 & 2 & 3 \\
\hline 3 & 64 & $\mathrm{M}$ & Glioma IV & 27.2 & Frontal & No & 12.5 & 1 & 0 & 1 & 6 & 8 \\
\hline 4 & 69 & $\mathrm{~F}$ & Glioma IV & 16.1 & Temporal & Mild & 45 & 6 & 1 & 1 & 12 & 20 \\
\hline 5 & 36 & $\mathrm{~F}$ & Astrocytoma II & 1.4 & Temporal & No & 3.75 & 1 & 4 & 2 & 2 & 9 \\
\hline 6 & 56 & $\mathrm{~F}$ & Metastasis (breast) & 6.7 & Frontal & No & 25 & 0 & 0 & 1 & 8 & 9 \\
\hline 7 & 41 & M & Ependymoma III & 5.6 & Angular gyrus & No & 6.25 & 0 & 0 & 1 & 4 & 5 \\
\hline 8 & 38 & $\mathrm{~F}$ & Oligoastrocytoma III & 7 & Temporal & No & 27.5 & 0 & 4 & 1 & 7 & 12 \\
\hline 9 & 65 & $\mathrm{~F}$ & Glioma IV & 31.1 & Temporal & Mild & 40 & 1 & 1 & 2 & 8 & 12 \\
\hline 10 & 38 & M & Glioma III & 21.9 & Frontal & No & 2.5 & 0 & 1 & 0 & 4 & 5 \\
\hline 11 & 29 & $\mathrm{~F}$ & Glioma III & 41.7 & Frontal & No & 12.5 & 2 & 5 & 0 & 5 & 12 \\
\hline 12 & 35 & M & Oligoastrocytoma III & 7.7 & Temporal & No & 7.5 & 1 & 3 & 2 & 8 & 14 \\
\hline 13 & 36 & M & Ganglioglioma & 0.7 & Temporal & No & 0 & 0 & 1 & 1 & 1 & 3 \\
\hline 14 & 59 & M & Lymphoma & 6.4 & Parietal & No & 13.75 & 1 & 2 & 2 & 3 & 8 \\
\hline 15 & 33 & M & Glioma III & 74.1 & Temporal & No & 5 & 0 & 3 & 0 & 6 & 9 \\
\hline 16 & 38 & M & Glioma III & 9.4 & Temporal & No & 8.75 & 0 & 0 & 1 & 11 & 12 \\
\hline 17 & 21 & M & Glioma IV & 58 & Frontal & No & 7.5 & 0 & 3 & 2 & 9 & 14 \\
\hline 18 & 39 & $\mathrm{~F}$ & Glioma III & 41.1 & Parietal & No & 10 & 0 & 1 & 1 & 14 & 16 \\
\hline 19 & 60 & $\mathrm{M}$ & Glioma IV & 24.3 & Temporal & No & 41.25 & 2 & 1 & 0 & 2 & 5 \\
\hline 20 & 45 & $\mathrm{~F}$ & Glioma II & 67.9 & Temporal & Mild & 7.5 & 0 & 1 & 0 & 3 & 4 \\
\hline 21 & 49 & M & Glioma II & 0.73 & Parietal & No & 2.5 & 0 & 0 & 1 & 2 & 3 \\
\hline 22 & 57 & $\mathrm{~F}$ & Glioma III & 2.9 & Frontal & No & 11.25 & 2 & 1 & 0 & 5 & 8 \\
\hline 23 & 35 & $\mathrm{~F}$ & Cavernoma & 0.44 & Temporal & No & 3.75 & 0 & 2 & 1 & 0 & 3 \\
\hline 24 & 50 & $\mathrm{~F}$ & Glioma III & 10.5 & Frontal & No & 20 & 0 & 0 & 0 & 2 & 2 \\
\hline 25 & 29 & $\mathrm{M}$ & Glioma II & 26.8 & Frontal & No & 17.5 & 14 & 4 & 3 & 1 & 22 \\
\hline 26 & 66 & M & Metastasis (kidney) & 1.98 & Opercular & No & 20 & 0 & 4 & 8 & 2 & 14 \\
\hline 27 & 68 & M & Glioma III & 3 & Temporal & No & 11.25 & 0 & 5 & 1 & 6 & 12 \\
\hline 28 & 52 & $\mathrm{~F}$ & Glioma III & 36 & Temporal & No & 15 & 0 & 0 & 5 & 3 & 8 \\
\hline 29 & 52 & M & Meningioma & 32.9 & Frontal & No & 18.75 & 2 & 1 & 0 & 4 & 7 \\
\hline 30 & 54 & $\mathrm{M}$ & Glioma IV & 42.8 & Temporal & Mild & 16.25 & 2 & 2 & 0 & 6 & 10 \\
\hline Total & & & & & & & & $35(13 \%)$ & $53(19 \%)$ & $37(13 \%)$ & $151(55 \%)$ & $276(100 \%)$ \\
\hline
\end{tabular}

Baseline error rate = relative frequency of wrong or inconsistent picture naming during the baseline object-naming task; $\mathrm{H}=$ hesitation; $\mathrm{NR}=$ no response, $\mathrm{P}=$ performance error; $p t=$ patient; $S=$ semantic paraphrasis.

tions, 1) tractogram in tumor proximity and 2) individual positive rTMS spot within projected craniotomy $(n=364)$, $77.4 \%$ resulted in successful FT (Table 4). Tracking success was most likely for DTI-FT of rTMS spots with the error category "hesitation" (84.5\%). In contrast, the use of "no response" rTMS spots led to successful FT in 67.2\% of cases $(C C=0.161 ; p=0.019)$. When analyzing the different tractogram ROIs, the use of the AF tractogram resulted most frequently in a successful tracking result (CC $=0.158 ; \mathrm{p}=0.054$ ).

\section{Expert Evaluations}

A total of 135 case evaluations were analyzed (Fig. 4). The landmark-based approaches (Ia and Ib) were rated best regarding their usefulness for surgical planning. In contrast, the other approaches (IIa, IIb, III) were less frequently evaluated as very useful/useful and instead were most often not considered useful. A "disturbing" impact on the spatial analysis between the language network and the tumor, such as visualized tracts showing an abnormal course with a high proportion of aberrant fibers, which can complicate the evaluator's interpretation of which parts are eloquent, was rarely assessed but most likely to occur with the rTMS-based (IIa) and rTMS-enhanced (III) approaches. The subcategory of usefulness for risk assessment was rated with similar distributions, showing the best results for the lesion-focused landmark-based approach (Ib). Again, best accuracy ratings were achieved by the landmark-based approaches (highly/mostly accurate: Ia, $87.5 \%$; Ib, 90.2\%), whereas mostly inaccurate/completely inaccurate ratings were more often obtained for the other approaches (IIa, IIb, and III). However, about half of the cases of the postprocessed rTMS-based approach (IIb) (49.5\%) and of the rTMS-enhanced approach (III) (49.1\%) 
also got highly/mostly accurate ratings. The perceived information content was assessed as high or balanced in $65.9 \%$ or $22.2 \%$ of cases of the landmark-based approach (Ia). After focusing only on tracts in tumor proximity (Ib), a shift from a high (39.3\%) to a balanced (48.1\%) perceived information content was observed. The other approaches were rated very heterogeneously regarding their perceived information content. The rTMS-based tractography (IIa) resulted in cluttered perceived information content in $34.1 \%$ of cases, which could be improved by removal of aberrant fibers (IIb).

Finally, the lesion-focused landmark-based approach (Ib) was the most preferred method for $39.8 \%$ of the raters, followed by the rTMS-enhanced method (III) (21.8\%; Fig. 4). In contrast, the less frequently preferred method was the rTMS-based approach without postprocessing (IIa) $(5.2 \%)$.

\section{Discussion}

In this study, we compared different FT protocols in their ability to visualize five principal language tracts. Our results clearly indicate that, even in the altered anatomy of brain tumor patients, landmark-based tractography (Ia, Ib) presents a robust technique for delineation of principal language tracts, with the best ratings for surgical planning, risk assessment, perceived accuracy, and perceived information content. In contrast, DTI-FT based purely on positive rTMS spots (IIa, IIb) did not reliably accomplish visualization, and the resulting tractograms were smaller in volume. The lesion-focused landmark-based tracking (Ib) was the overall preferred method, with the highest scores in usefulness and information content balance.

\section{Transcranial Magnetic Stimulation}

Transcranial magnetic stimulation (TMS) presents a noninvasive, electrophysiological technique which can induce strong, short-lasting electric fields that alter corticoneural excitability ${ }^{24}$ and can be aided by the implementation of a stereotactic navigation system allowing precise mapping of cortical areas. ${ }^{25} \mathrm{~A}$ recent meta-analysis confirmed the usefulness of TMS motor mapping in clinical routine showing reduced number of postoperative motor deficits and increased rate of gross-total resections in patients with brain tumors affecting the motor cortex or the corticospinal tract. ${ }^{26}$ TMS language mapping protocols utilize repetitive TMS pulses in order to disrupt signaling in targeted areas. This temporary inhibition of languageinvolved cortical regions impairs performance in concurrently conducted language tasks, following the approach of DCS during awake craniotomy. Different comparative studies with DCS showed a varying specificity of language-positive spots $(35.6 \%-90 \%)$, but there is evidence of a high negative predictive value (83.9\%-99\%). ${ }^{7-9}$ In this study, the number of positive rTMS spots as well as the correlation between them and the baseline error rate were comparable to those reported in the literature..$^{15,27}$ While the exact mechanisms through which rTMS disrupts language processing are as yet elusive, protocol refinements are ongoing. However, we followed a standardized core protocol for rTMS language mapping that was

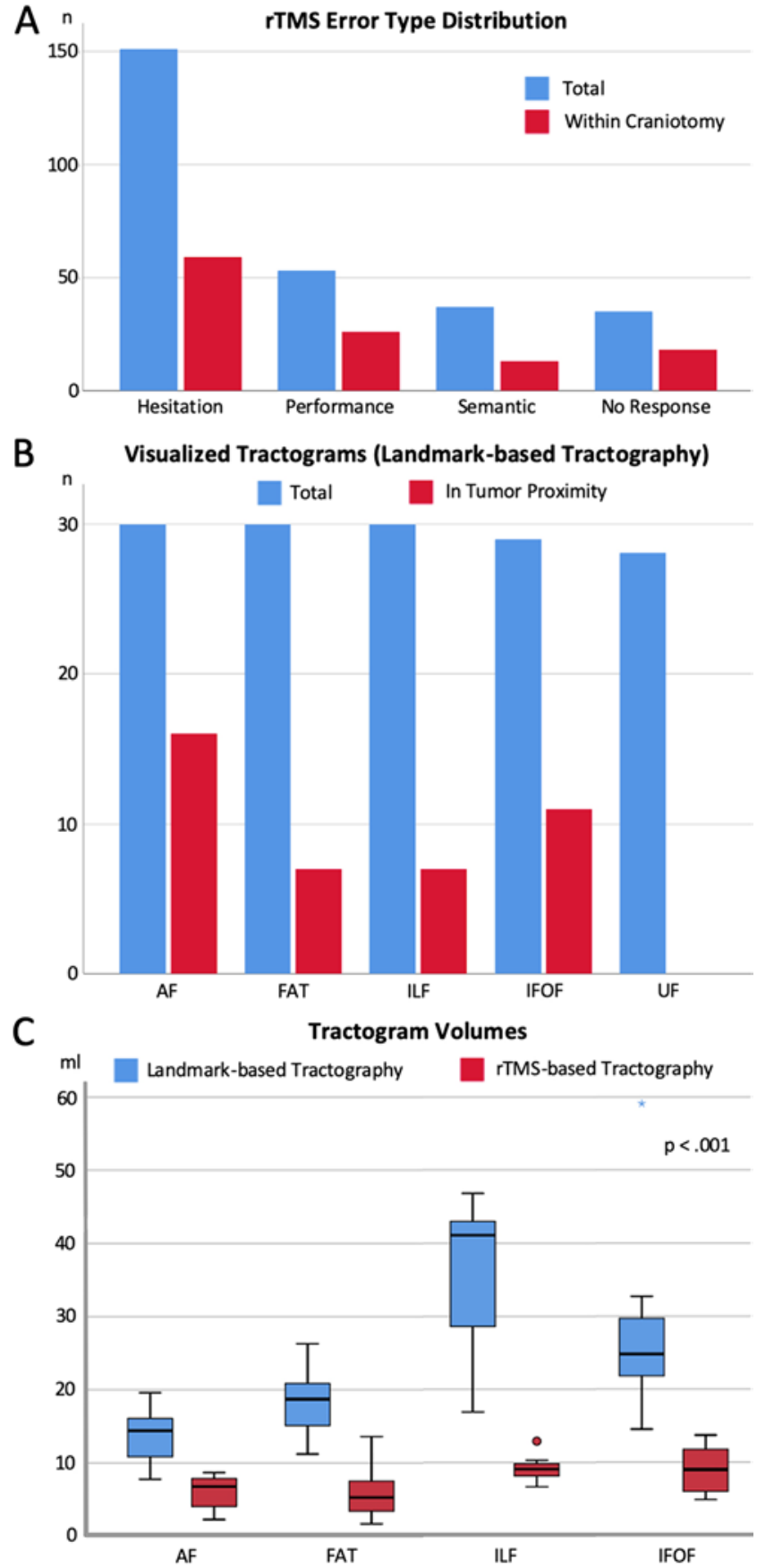

FIG. 3. rTMS error type distribution (A), visualized tracts for the landmark-based approach in total (la) and in tumor proximity (tumor-totract distance $\leq 20 \mathrm{~mm})(\mathrm{lb})(\mathrm{B})$, and comparison of tract volumes of the landmark-based (la) and rTMS-based (Ilb) tractography (C). Figure is available in color online only.

agreed upon in a recent expert panel..$^{20}$ Functional MRI (fMRI) offers another alternative method for noninvasive localization of function. However, navigated TMS (nTMS) was found to be superior in the identification of seeding regions for tractography of the corticospinal $\operatorname{tract}^{28}$ and lacks some important limitations that arise with fMRI in tumor patients. ${ }^{29}$ In detail, tumor-associated neurovascu- 
TABLE 3. Comparison of landmark-based (la) and nTMS-based (Illb) tractography

\begin{tabular}{lccccc}
\hline & AF & FAT & ILF & IFOF & UF \\
\hline Successful tracking w/ landmark-based tractography (la) & $30(100 \%)$ & $30(100 \%)$ & $30(100 \%)$ & $29(96.7 \%)$ & $28(93.3 \%)$ \\
\hline Successful tracking w/ nTMS-based tractography (Ilb) & $7(23.3 \%)$ & $16(53.5 \%)$ & $7(23.3 \%)$ & $11(36.7 \%)$ & 0 \\
\hline High vol & $2(28.5 \%)$ & $1(6.3 \%)$ & $1(14.3 \%)$ & 0 & 0 \\
\hline Intermediate vol & $3(43 \%)$ & $3(18.2 \%)$ & $2(28.6 \%)$ & $5(45.5 \%)$ & 0 \\
\hline Low vol & $2(28.5 \%)$ & $12(75 \%)$ & $4(57.1 \%)$ & $6(54.5 \%)$ & 0 \\
\hline
\end{tabular}

High volume, $\geq 66 \%$ of landmark-based tractograms; intermediate volume, $33 \%-66 \%$ of landmark-based tractograms; low volume, $\leq 33 \%$ of landmark-based tractograms.

lar uncoupling and impaired oxygenation can compromise the analysis of eloquent brain regions. ${ }^{30,31}$ Thus, nTMS is the preferred noninvasive mapping technique for surgical oncology according to a recent review of the literature. ${ }^{30}$

\section{Language Network}

Traditional models of language are based on the paradigm of specific cortical regions harboring specific functions. Accumulating evidence introduced a shift of understanding toward a hodotopical view, in which language results from a complex cortico-subcortical network., ${ }^{1,2}$ Two main pathways in this network can be differentiated: the phonological dorsal pathway (sensorimotor integration) and the semantic ventral pathway. ${ }^{5}$ Part of the dorsal pathway is served by the AF, whose interruption is associated with conduction aphasia. ${ }^{32,33}$ Subcortical stimulation of the $\mathrm{AF}$ during awake surgery reliably resulted in a speech arrest and phonological paraphasia. ${ }^{34}$ Disruption of the IFOF, which enables the direct ventral pathway by connecting the posterior occipital lobe to the anterior cortical frontal areas, reproducibly elicits semantic paraphrasias. ${ }^{32}$ DTI imaging suggests that the ILF plays a substantial role in the process of object recognition. ${ }^{35}$ The bidirectional UF, which connects the anterior temporal lobe to the lateral orbitofrontal cortex, is considered an important pathway for language processing and semantic retrieval. ${ }^{36}$ Interestingly, the resection of the temporal pole (e.g., during epilepsy surgeries) does not lead to a new aphasia if the temporal stem (especially the IFOF) is preserved. ${ }^{37}$ The FAT connects parts of the inferior and superior frontal gyri to the anterior cingulate cortex. Abnormalities in this more recently described pathway are associated with an impairment of articulation processing ${ }^{38}$ but are often reversible. ${ }^{39}$

Numerous other studies have contributed to the rapidly evolving understanding of these tracts in language function and their respective functional roles. ${ }^{23,32}$ As damage to these subcortical association fibers is associated with marked and irreversible deficits, the importance of their preservation during tumor surgery is paramount. ${ }^{4}$

\section{DTI-FT Approaches}

DTI was first proposed in 1994 by Peter Basser ${ }^{40}$ as a technology to reveal subcortical white matter architecture. By measuring the diffusion coefficient of water during MRI, DTI allows for the estimation of white matter tract trajectories through numerous methods such as the deterministic FACT (fiber assignment by continuous tracking) and TEND (tensor deflection) algorithms, ${ }^{22}$ which were employed in our study. Today DTI presents a valuable asset for clinical routine and research.

Delineation of specific language fascicles can be achieved by providing ROIs as seeding regions in anatomically defined regions. A recent study provided an atlas of anatomical landmarks which can be used as ROIs to reliably visualize the principal language tracts in a user-independent way. ${ }^{11}$ The tracking results of this study confirmed its reliability, with highest tracking success for the landmark-based approaches (Ia, Ib). In contrast, the rTMS-based approaches (IIa, IIb) were only effective to visualize one of five principal tracts in the median. Although the postprocessing (IIb) helped to improve the perceived accuracy and the perceived information content, no relevant improvement for surgical planning or risk assessment could be obtained. Thus, tracking protocols based on rTMS ROI seeding alone cannot be recommended for presurgical assessment of left-sided perisylvian tumors. The analysis of the expert evaluations highlighted this, with a preference for the lesion-focused landmark-based approach (Ib) for all qualities studied.

The purely landmark-based approaches, however, do not directly reflect function, which can potentially deviate in the altered anatomy of tumor patients. For this purpose,

TABLE 4. Frequency of successful rTMS-enhanced tractography (III)

\begin{tabular}{ccccccc}
\hline & \multicolumn{5}{c}{ Tract } & \multirow{2}{*}{ Total } \\
\cline { 2 - 6 } rTMS Error Category & AF & FAT & UF & ILF & IFOF & $84.5 \%$ \\
\hline Hesitation & $89.4 \%$ & $71.9 \%$ & $80 \%$ & $90 \%$ & $87.5 \%$ & $87.2 \%$ \\
\hline No response & $70.6 \%$ & $73.3 \%$ & $75 \%$ & $20 \%$ & $64.3 \%$ & $67.6 \%$ \\
\hline Performance & $90.5 \%$ & $50 \%$ & $71.4 \%$ & $93.3 \%$ & $70.6 \%$ & $75.6 \%$ \\
\hline Semantic & $75 \%$ & $71.4 \%$ & $62.5 \%$ & $87.5 \%$ & $61.1 \%$ & $71.7 \%$ \\
\hline Total & $84.6 \%$ & $67.1 \%$ & $73.8 \%$ & $84.5 \%$ & $81.1 \%$ & $77.4 \%$ \\
\hline
\end{tabular}


A

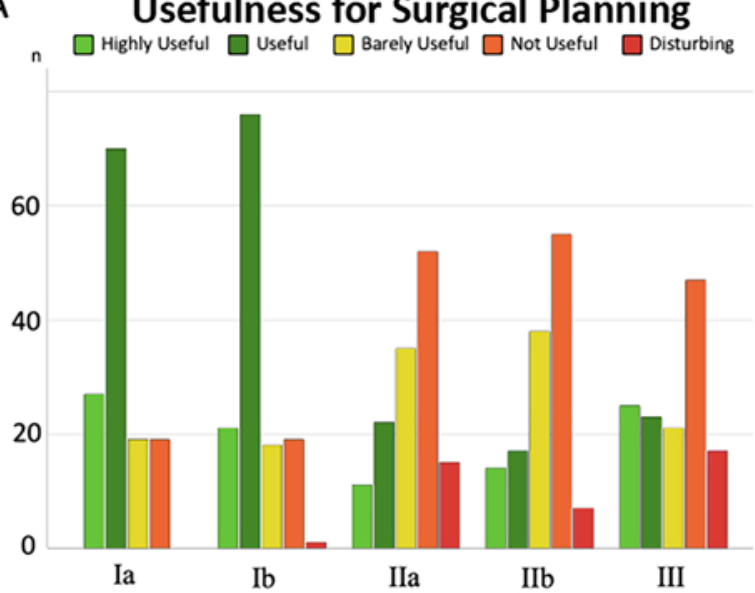

C Perceived Accuracy of Tractograms

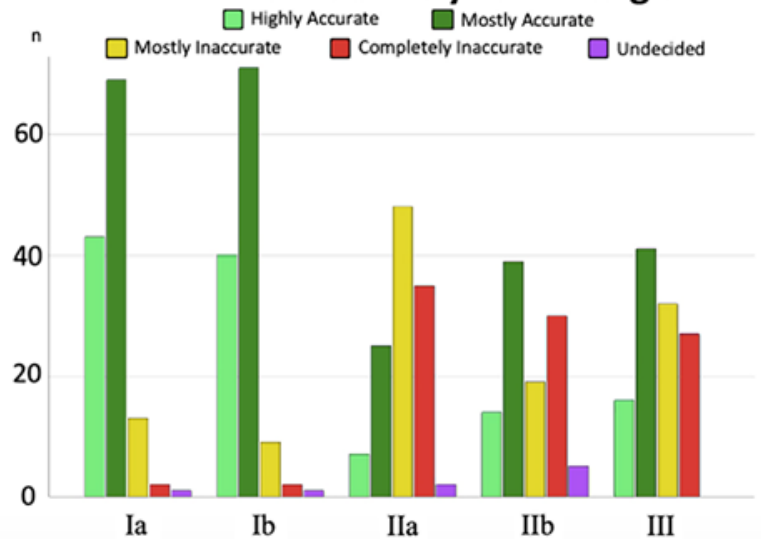

Ia

$E^{n}$

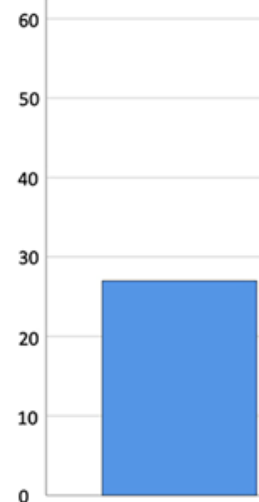

Ia

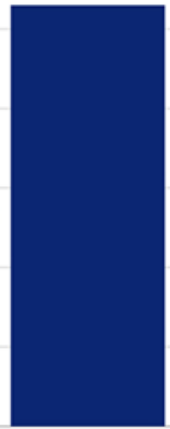

$\mathrm{Ib}$
Preferred Method

\section{B Usefulness for Risk Assessment}

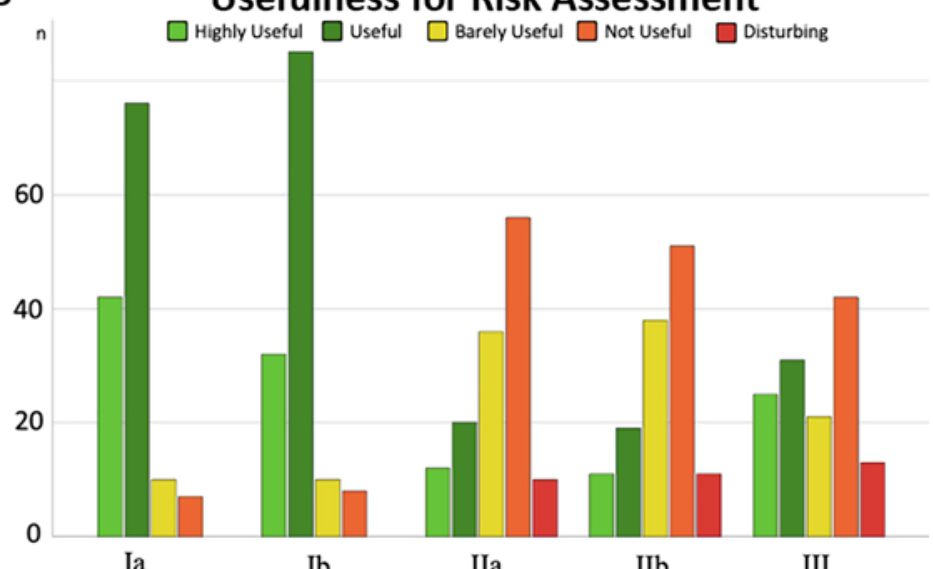

Ia

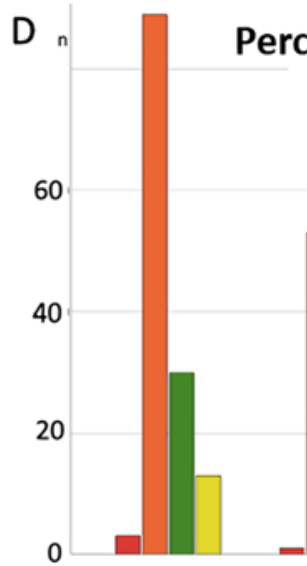

Ia
$\mathrm{Ib}$

IIb

III

Perceived Information Content
$\mathrm{Ib}$

$\square$ Balanced $\square$ Low $\square$ Undecided

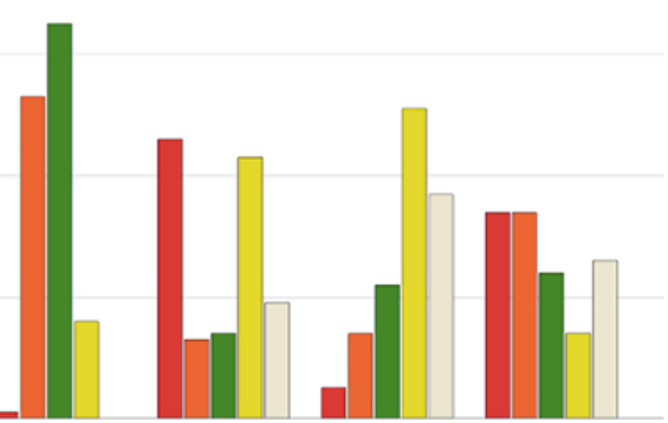

IIa

IIb

III

FIG. 4. Bar charts of 135 expert evaluations depending on the different algorithms: landmark-based (la), landmark-based (lesionfocused) (Ib), rTMS-based (Ila), rTMS-based postprocessed (IIb), and rTMS-enhanced (III) approaches. Various criteria were assessed: usefulness for surgical planning (A), usefulness for risk assessment (B), perceived accuracy of tractograms (C), perceived information content (D), and overall preferred method (E). Figure is available in color online only.

various approaches to carry out DTI-FT with integration of functional data from rTMS language mapping have been described. Protocols with enlarged rTMS-positive spots (up to 5-mm radius) were tested with variations of tracking parameters such as the individual FA or the minimum fiber length. ${ }^{4,12,13,41-43}$ In addition to our study results, there is further evidence that-even with individual FA
thresholds-rTMS spots alone are not sufficient to reliably visualize language tracts ${ }^{41,42}$ resulting in low ratings for clinical usability. One can consider different causes and reasons: 1) DTI estimates white matter tracts by calculating the diffusion coefficients. At the cortical level, where the rTMS spots are located, the diffusion coefficients are very low, which affects the tracking results. 2) Regarding 
the low positive predictive value of current rTMS language mapping protocols, ranging between $16 \%$ and $69 \%, 7,46,47$ most rTMS-positive spots are false positive, leading to the visualization of aberrant and unrelated pathways. 3) In many studies, ROIs were defined by rTMS-positive spots enlarged to a radius of $5 \mathrm{~mm}$. This leads to a large ROI area, which may increase the nonspecificity.

Despite these limitations, function-derived ROIs theoretically bear the potential for fully automated and functionally informed language tractography, with the advantage that ROI seeding does not require expert skills or neuroanatomical knowledge.

In consequence, an approach combining functional and tractography-derived ROIs to visualize the cortico-subcortical connections (III) was first proposed by Raffa et al. in 2017. ${ }^{44}$ This tracking technique is especially suited to visualize specific tracts connecting landmark-based subcortical tractograms with cortical-located rTMS-positive language spots. In our study, we observed a trend such that more cortico-subcortical connections could be revealed from rTMS spots with error category hesitation and located in the AF. This innovative approach was positively evaluated by the experts in our survey, with the rTMS-enhanced approach (III) being the second overall preferred method. Interestingly, the best tracking results for visualization of the corticospinal tract were also achieved by combining TMS-based and landmark-based ROIs. ${ }^{45}$ As a limitation of this technique, it must be clearly stated that the functional relevance of these cortico-subcortical connections has not yet been analyzed.

Future studies are recommended in which innovative techniques such as spherical deconvolution tractography and machine learning-based approaches for automatic data processing may be combined with high-quality raw data acquisitions.

\section{Study Limitations}

Deterministic DTI has methodological limitations, as crossing fibers and the presence of edema may induce aberrant fiber reconstruction. We have used tracking parameters based on all published standards of rTMS-based FTs. However, we did not employ individual threshold values, as suggested in previous works with varying success. ${ }^{12,41}$ Further studies are needed to investigate potential impacts on clinical outcome, as well as correlations with intraoperative DCS. However, even with DCS as the gold standard, rTMS and DTI data should be considered valuable and relevant, because not all patients are candidates for awake craniotomy. The tractography results were deemed useful for presurgical planning, thus potentially altering the surgical strategy or decision making. More data are needed to meaningfully reveal potential patterns in connectivity between specific error types and tracts. Furthermore, tract fragmentation might be required to distinguish between individual functional streams.

\section{Conclusions}

The lesion-focused landmark-based approach (Ib) achieved the best ratings and enabled visualization of the principal language tracts in almost all cases. Our results underscore the reliability and usability of landmark-based language tractography for surgical planning in tumor patients. Due to the inability to reliably visualize the subcortical language network, tracking algorithms using rTMS spots as ROIs alone (IIa, IIb) are of questionable clinical use. The rTMS-enhanced approach (III) was appreciated by the experts because it revealed cortico-subcortical connections, but the functional relevance of these is still unclear. Further studies are urgently indicated to investigate the validity of the different language tractography algorithms.

\section{Acknowledgments}

The authors acknowledge the support of the Cluster of Excellence "Matters of Activity. Image Space Material" funded by the Deutsche Forschungsgemeinschaft (DFG; German Research Foundation) under Germany's Excellence Strategy_EXC 2025.

Dr. Rosenstock is a participant in the BIH Charite Junior Digital Clinician Scientist Program funded by the Charité - Universitätsmedizin Berlin and the Berlin Institute of Health.

\section{References}

1. Tremblay P, Dick AS. Broca and Wernicke are dead, or moving past the classic model of language neurobiology. Brain Lang. 2016;162:60-71.

2. Hickok G, Poeppel D. The cortical organization of speech processing. Nat Rev Neurosci. 2007;8(5):393-402.

3. Basilakos A, Fillmore PT, Rorden C, et al. Regional white matter damage predicts speech fluency in chronic post-stroke aphasia. Front Hum Neurosci. 2014;8:845.

4. Negwer C, Beurskens E, Sollmann N, et al. Loss of subcortical language pathways correlates with surgery-related aphasia in patients with brain tumor: an investigation via repetitive navigated transcranial magnetic stimulation-based diffusion tensor imaging fiber tracking. World Neurosurg. 2018;111:e806-e818.

5. Chang EF, Raygor KP, Berger MS. Contemporary model of language organization: an overview for neurosurgeons. $J$ Neurosurg. 2015;122(2):250-261.

6. Rahimpour S, Haglund MM, Friedman AH, Duffau H. History of awake mapping and speech and language localization: from modules to networks. Neurosurg Focus. 2019;47(3):E4.

7. Tarapore PE, Findlay AM, Honma SM, et al. Language mapping with navigated repetitive TMS: proof of technique and validation. Neuroimage. 2013;82:260-272.

8. Bährend I, Muench MR, Schneider H, et al. Incidence and linguistic quality of speech errors: a comparison of preoperative transcranial magnetic stimulation and intraoperative direct cortex stimulation. J Neurosurg. Published online May 29, 2020. doi:10.3171/2020.3.JNS193085

9. Kawashima A, Krieg SM, Faust K, et al. Plastic reshaping of cortical language areas evaluated by navigated transcranial magnetic stimulation in a surgical case of glioblastoma multiforme. Clin Neurol Neurosurg. 2013;115(10):2226-2229.

10. Ille S, Sollmann N, Butenschoen VM, et al. Resection of highly language-eloquent brain lesions based purely on rTMS language mapping without awake surgery. Acta Neurochir (Wien). 2016;158(12):2265-2275.

11. Fekonja L, Wang Z, Bährend I, et al. Manual for clinical language tractography. Acta Neurochir (Wien). 2019;161(6): $1125-1137$.

12. Sollmann N, Kubitscheck A, Maurer S, et al. Preoperative language mapping by repetitive navigated transcranial magnetic stimulation and diffusion tensor imaging fiber tracking and their comparison to intraoperative stimulation. Neuroradiology. 2016;58(8):807-818.

13. Sollmann N, Negwer C, Tussis L, et al. Interhemispheric 
connectivity revealed by diffusion tensor imaging fiber tracking derived from navigated transcranial magnetic stimulation maps as a sign of language function at risk in patients with brain tumors. J Neurosurg. 2017;126(1):222-233.

14. O'Brien BC, Harris IB, Beckman TJ, et al. Standards for reporting qualitative research: a synthesis of recommendations. Acad Med. 2014;89(9):1245-1251.

15. Schwarzer V, Bährend I, Rosenstock T, et al. Aphasia and cognitive impairment decrease the reliability of rnTMS language mapping. Acta Neurochir (Wien). 2018;160(2):343-356.

16. Huber W, Weniger D, Poeck K, Willmes K. The Aachen Aphasia Test rationale and construct validity (author's transl). Article in German. Nervenarzt. 1980;51(8):475-482.

17. Oldfield RC. The assessment and analysis of handedness: the Edinburgh inventory. Neuropsychologia. 1971;9(1):97-113.

18. Rossini PM, Barker AT, Berardelli A, et al. Non-invasive electrical and magnetic stimulation of the brain, spinal cord and roots: basic principles and procedures for routine clinical application. Report of an IFCN committee. Electroencephalogr Clin Neurophysiol. 1994;91(2):79-92.

19. Rothwell JC, Hallett M, Berardelli A, et al. Magnetic stimulation: motor evoked potentials. Electroencephalogr Clin Neurophysiol Suppl. 1999;52:97-103.

20. Krieg SM, Lioumis P, Mäkelä JP, et al. Protocol for motor and language mapping by navigated TMS in patients and healthy volunteers; workshop report. Acta Neurochir (Wien). 2017;159(7):1187-1195.

21. Gerhardt J, Sollmann N, Hiepe P, et al. Retrospective distortion correction of diffusion tensor imaging data by semielastic image fusion-evaluation by means of anatomical landmarks. Clin Neurol Neurosurg. 2019;183:105387.

22. Mori S, Crain BJ, Chacko VP, van Zijl PC. Three-dimensional tracking of axonal projections in the brain by magnetic resonance imaging. Ann Neurol. 1999;45(2):265-269.

23. Catani M, Thiebaut de Schotten M. A diffusion tensor imaging tractography atlas for virtual in vivo dissections. Cortex. 2008;44(8):1105-1132.

24. Barker AT, Jalinous R, Freeston IL. Non-invasive magnetic stimulation of human motor cortex. Lancet. 1985;1(8437): 1106-1107.

25. Rosenstock T, Grittner U, Acker G, et al. Risk stratification in motor area-related glioma surgery based on navigated transcranial magnetic stimulation data. J Neurosurg. 2017;126(4): 1227-1237.

26. Raffa G, Scibilia A, Conti A, et al. The role of navigated transcranial magnetic stimulation for surgery of motor-eloquent brain tumors: a systematic review and meta-analysis. Clin Neurol Neurosurg. 2019;180:7-17.

27. Rösler J, Niraula B, Strack V, et al. Language mapping in healthy volunteers and brain tumor patients with a novel navigated TMS system: evidence of tumor-induced plasticity. Clin Neurophysiol. 2014;125(3):526-536.

28. Weiss Lucas C, Tursunova I, Neuschmelting V, et al. Functional MRI vs. navigated TMS to optimize M1 seed volume delineation for DTI tractography. A prospective study in patients with brain tumours adjacent to the corticospinal tract. Neuroimage Clin. 2016;13:297-309.

29. Tarapore PE, Tate MC, Findlay AM, et al. Preoperative multimodal motor mapping: a comparison of magnetoencephalography imaging, navigated transcranial magnetic stimulation, and direct cortical stimulation. J Neurosurg. 2012; 117(2):354-362.

30. Sanai N, Berger MS. Surgical oncology for gliomas: the state of the art. Nat Rev Clin Oncol. 2018;15(2):112-125.

31. Freyschlag CF, Krieg SM, Kerschbaumer J, et al. Imaging practice in low-grade gliomas among European specialized centers and proposal for a minimum core of imaging. $\mathrm{J} \mathrm{Neu-}$ rooncol. 2018;139(3):699-711.

32. Duffau H. Stimulation mapping of white matter tracts to study brain functional connectivity. Nat Rev Neurol. 2015; 11(5):255-265.

33. Sarubbo S, De Benedictis A, Merler S, et al. Towards a functional atlas of human white matter. Hum Brain Mapp. 2015; 36(8):3117-3136.

34. Ries SK, Piai V, Perry D, et al. Roles of ventral versus dorsal pathways in language production: An awake language mapping study. Brain Lang. 2019;191:17-27.

35. Ortibus E, Verhoeven J, Sunaert S, et al. Integrity of the inferior longitudinal fasciculus and impaired object recognition in children: a diffusion tensor imaging study. Dev Med Child Neurol. 2012;54(1):38-43.

36. Von Der Heide RJ, Skipper LM, Klobusicky E, Olson IR. Dissecting the uncinate fasciculus: disorders, controversies and a hypothesis. Brain. 2013;136(Pt 6):1692-1707.

37. de Souza JPSAS, Ayub G, Nogueira M, et al. Temporopolar amygdalohippocampectomy: seizure control and postoperative outcomes. J Neurosurg. Published online May 15, 2020. doi:10.3171/2020.3.JNS192624

38. Catani M, Mesulam MM, Jakobsen E, et al. A novel frontal pathway underlies verbal fluency in primary progressive aphasia. Brain. 2013;136(Pt 8):2619-2628.

39. Dick AS, Garic D, Graziano P, Tremblay P. The frontal aslant tract (FAT) and its role in speech, language and executive function. Cortex. 2019;111:148-163.

40. Basser PJ, Mattiello J, LeBihan D. MR diffusion tensor spectroscopy and imaging. Biophys J. 1994;66(1):259-267.

41. Sollmann N, Negwer C, Ille S, et al. Feasibility of nTMSbased DTI fiber tracking of language pathways in neurosurgical patients using a fractional anisotropy threshold. J Neurosci Methods. 2016;267:45-54.

42. Negwer C, Sollmann N, Ille S, et al. Language pathway tracking: comparing nTMS-based DTI fiber tracking with a cubic ROIs-based protocol. J Neurosurg. 2017;126(3):10061014.

43. Negwer C, Ille S, Hauck T, et al. Visualization of subcortical language pathways by diffusion tensor imaging fiber tracking based on rTMS language mapping. Brain Imaging Behav. 2017;11(3):899-914.

44. Raffa G, Bährend I, Schneider H, et al. A novel technique for region and linguistic specific nTMS-based DTI fiber tracking of language pathways in brain tumor patients. Front Neurosci. 2016;10:552.

45. Rosenstock T, Giampiccolo D, Schneider H, et al. Specific DTI seeding and diffusivity-analysis improve the quality and prognostic value of TMS-based deterministic DTI of the pyramidal tract. Neuroimage Clin. 2017;16:276-285.

46. Bahrend I, Muench MR, Schneider $\mathrm{H}$, et al. Incidence and linguistic quality of speech errors: a comparison of preoperative transcranial magnetic stimulation and intraoperative direct cortex stimulation. J Neurosurg. 2021;134(5):1409-1418.

47. Tarapore PE, Findlay AM, Honma SM, et al. Language mapping with navigated repetitive TMS: proof of technique and validation. Neuroimage. 2013;82:260-272.

\section{Disclosures}

The authors report no conflict of interest concerning the materials or methods used in this study or the findings specified in this paper.

\section{Author Contributions}

Conception and design: Rosenstock, Picht. Acquisition of data: Rosenstock, Silva, Tuncer, Picht. Analysis and interpretation of data: Rosenstock, Silva, Tuncer, Picht. Drafting the article: Rosenstock, Silva. Critically revising the article: Rosenstock, Tuncer, Vajkoczy, Picht. Reviewed submitted version of manuscript: all authors. Approved the final version of the manuscript on behalf of all authors: Rosenstock. Statistical 
analysis: Rosenstock, Silva. Administrative/technical/material support: Vajkoczy, Picht. Study supervision: Rosenstock,

Vajkoczy, Picht.

\section{Supplemental Information}

Online-Only Content

Supplemental material is available with the online version of the article.

Supplemental Material. https://thejns.org/doi/suppl/10.3171/ 2020.12.JNS204028.

\section{Correspondence}

Tizian Rosenstock: Charité - Universitätsmedizin Berlin, corporate member of Freie Universität Berlin and Humboldt-Universität zu Berlin, Berlin, Germany. tizian.rosenstock@charite.de. 